\title{
ESTRATEGIAS PARA LA PUESTA EN VALOR DEL SITIO ARQUEOLÓGICO CANTAMARCA, PARA EL DESARROLLO DEL TURISMO SOSTENIBLE EN LA PROVINCIA DE CANTA
}

\author{
STRATEGIES FOR VALUE ENHANCEMENT OF THE ARCHAEOLOGICAL SITE "CANTAMARCA" \\ DEVELOPMENT OF SUSTAINABLE TOURISM IN THE PROVINCE CANTA
}

Niko Cruz Gonzales*

nikturismo@hotmail.com

\begin{abstract}
RESUMEN
La investigación realizada por los miembros del equipo: Turismo hacia el Bicentenario del sitio arqueológico Cantamarca (perteneciente a la provincia de Canta) desarrolló un diagnóstico situacional a fin de plantear los procesos y estrategias para desarrollar la puesta en valor del sitio arqueológico. Cantamarca proviene de dos palabras: la primera, "Canta”, era el nombre del pueblo que habitó en este sitio, y "marca”, que quiere decir "pueblo" o "lugar". A su vez la primera palabra "Canta" es atribuida a los grandes cazadores de vicuña, por lo que el nombre final del complejo deviene en la traducción de "Pueblo de grandes cazadores de vicuña". Según la historiadora Dra. María Rostworowski Tovar y el Arqul. Carlos Arturo Farfán Lobatón, Cantamarca fue construida durante el Intermedio tardío (1100 d.C.) y sobrevivió a la conquista de los Incas (1450), quienes la reorganizaron y ampliaron hasta el año de 1550, cuando los nuevos conquistadores españoles reubicaron a sus pobladores en la actual ciudad de Canta, siendo Cantamarca abandonada definitivamente. A partir de un somero reconocimiento del lugar, describen sus características arquitectónicas, destacando los siguientes grupos: A) la muralla de circunvalación; B) el acueducto; C) las construcciones incaicas o “Tampus”; D) las construcciones preincaicas; E) la "Pucará" o Atalaya; F) la población militar o casas- tumbas; G) los fuertes de contención; H) las cavernas sepulcrales.

El sitio arqueológico de Cantamarca fue declarado Patrimonio Cultural de la Nación mediante Resolución Directoral Nacional $\mathrm{N}^{\circ}$ 051/INC año 2006, para su conservación, preservación y promoción como destino turístico principal de la provincia. La importancia del presente proyecto de investigación constituye el primer esfuerzo, a nivel de la provincia de Canta, por recuperar un patrimonio cultural, desarrollado mediante acciones priorizadas y conjuntas, a fin de brindar a la población nuevas alternativas de desarrollo sostenible, a nivel turístico, social, cultural y económico.

Palabras claves: Sitio arqueológico. estrategias, puesta en valor, desarrollo sostenible, turismo.
\end{abstract}

\section{ABSTRACT}

Research by team members: "Tourism towards the Bicentennial" Cantamarca Archaeological Site (located in the province of Canta) developing a situational analysis in order to raise the processes and strategies to develop the enhancement of the archaeological site. Cantamarca comes from two words, the first "Canta" was the name of the people who inhabited this place, and "marca", which

\footnotetext{
* Doctor en Derecho de la Universidad Nacional Federico Villarreal, magíster de Administración-Universidad Alas Peruanas, licenciado en Turismo-Universidad Nacional San Antonio Abad del Cusco, asesor de Proyectos de Inversión Publica y Privada del Sector Turismo
} 
means "people" or "place" turn the first word "Canta" is attributed the great hunters of vicuña, so the final name becomes complex in the translation of "People of great hunters vicuna."

According to historian Dr. Maria Tovar Rostworowski and Arqul. Carlos Arturo Farfán Lobatón mention that Cantamarca was built during the Late Intermediate Period (1100 AD) and survived the conquest of the Incas (1450), who the reorganized and expanded until 1550 when the new Spanish conquistadors relocated its residents in the present town of Canta, and finally abandoned Cantamarca. From your brief reconnaissance describes its architecture, particularly the following groups:

A) The wall ring, B) The aqueduct, C) or the Inca constructions "Tampus" the "Pucara" or Atalaya E), D) The pre-Inca constructions F) military town homes and graves; $G$ ) strong containment, $\mathrm{H}$ ) burial caves.

The archaeological site of Cantamarca, was declared Cultural Patrimony of the Nation by National Directorial Resolution No. 2006 051/INC for conservation, preservation and promotion as a top tourist destination of the province. The importance of this research project is the first effort at the level of the province of Canta regain a cultural heritage, prioritized and developed through joint actions, so in order to give people new options for sustainable development, both tourism, social, cultural and economic level.

Keywords: Archaeological site; strategies; value; sustainable development; tourism.

\section{PLANTEAMIENTO DEL PROBLEMA}

El Perú es uno de los focos a nivel mundial donde surgió una de las seis civilizaciones más complejas, cuya cumbre fue el Imperio del Tawantinsuyu. Como prueba de ello, existen decenas de miles de sitios arqueológicos que evidencian el alto grado de desarrollo cultural alcanzado, desarrollo truncado con la invasión hispana en 1532. Sin embargo, cada día, decenas de estos sitios son destruidos totalmente o mutilados, a fin de ampliar la frontera agrícola, construir obras productivas, extractivas o de servicios.

En los valles bajos y medios de los ríos Chancay, Chillón, Rímac y Lurín, se desenvolvió la sociedad Lima, entre los años 150 y 650 años d.C. Uno de ellos es el sitio arqueológico Cantamarca, que se ubica en la cuenca del río Chillón, en la margen izquierda, a $5 \mathrm{~km}$ al noroeste de la actual ciudad de Canta, con el punto Datum (PSAD-56) en las coordenadas UTM: Este: 327,320.2008 y Norte: 8'735,606.3528, a 3,759 m.s.n.m. en el cerro Cantamarca. Políticamente, se localiza en el distrito y provincia de Canta, departamento de Lima.

Cantamarca está construida en la cresta de un cerro escarpado, por lo que son pocos los espacios planos; sus edificios son circulares, con paredes y techos de piedras unidas con barro, sostenidos por una columna central. Para ampliar el espacio hábil se edificaron muchos terraplenes a diferentes niveles, ampliando de este modo el área construida.

Se divide en dos sectores (A-B) diferenciados entre sí por su organización espacial, componentes arquitectónicos y funcionalidad, así como su filiación cultural perteneciente al Intermedio tardío y Horizonte tardío.

\section{OBJETIVO}

Elaborar las estrategias para la recuperación cultural del sitio arqueológico de Cantamarca y la puesta en valor como destino turístico para mejorar la calidad de vida de la población local y de esta manera, contribuir al desarrollo socioeconómico, cultural y sostenible del distrito de Canta.

\section{JUSTIFICACIÓN}

La importancia del presente proyecto de investigación radica en que se constituye en el primer esfuerzo, a nivel de la provincia de Canta, por recuperar un patrimonio cultural, desarrollado mediante acciones priorizadas y conjuntas, a fin de lograr mejorar el estado socioeconómico y cultural de la población local. Salvo en el sitio arqueológico de Cantamarca, no se realizaron trabajos de restauración y conservación.

El proyecto es viable por cuanto ya se han realizado investigaciones, se han realizado algunos diagnósticos arqueológicos y sociológicos previos, y además se cuenta con el respaldo de la Municipalidad Provincial de Canta. Los profesionales que integran el presente proyecto de investigación cuentan con vasta experiencia en proyectos de desarrollo turístico sostenible.

Para la obtención de los objetivos y fines planteados, se cuenta con una metodología teórica y empíricamente sustentada. Los trabajos programados serán ejecutados siguiendo el cronograma señalado. El trabajo de campo consiste en la recolección de la información del sitio, mediante el registro sistemático y detallado de los materiales identificados. Posteriormente se realizará el trabajo de gabinete, donde se procesará toda la 
información recogida en el campo. La elaboración del informe final es la última etapa del proyecto. El desarrollo de todo el proyecto será siguiendo una metodología que será apoyada y preparada, según el marco teórico a utilizar, con base en la legislación vigente, esto es, el Reglamento de Investigaciones Arqueológicas y La Ley General de Patrimonio Cultural, tomando en consideración el Plan Estratégico de Desarrollo Turístico Nacional (PENTUR).

\section{HIPÓTESIS}

De acuerdo a los estudios realizados y la evaluación del potencial del sitio arqueológico de Cantamarca, se plantea la siguiente hipótesis:

Desarrollar el sitio arqueológico de Cantamarca como un producto turístico integral bajo criterios de sostenibilidad, con participación activa de la población, brindando una experiencia de calidad a los visitantes.

La recuperación del sitio arqueológico en el sector A y B logrará incrementar el flujo de visitantes nacionales y extranjeros. De igual manera, la recuperación del sector político administrativo de Cantamarca logrará cohesionar la identidad cultural de la población local.

El conocimiento de las características culturales de las sociedades que ocuparon este espacio geográfico en periodos prehispánicos y su difusión entre la población mediante publicaciones, conferencias y talleres creará una mayor interacción entre la población y su patrimonio cultural, lográndose finalmente la protección de estos.

Además, la recuperación de este extenso e imponente sitio arqueológico es óptimo para la generación del turismo en la localidad, pues la ubicación del pueblo de Canta, a treinta(30) minutos de la provincia de Canta, podría atraer a la población turística que viaja diariamente al destino turístico de Obrajillo a visitar Cantamarca, así como a desarrollar el turismo cultural y natural en la localidad.

Esto presupone el deseo de la población de recibir a los visitantes, la instalación de infraestructura de recepción de visitantes y la construcción de circuitos y paneles informativos, así como un museo de sitio en la localidad.

Estos logros traerán como resultado el factor de desarrollo turístico sostenible en la provincia de Canta, distritos y comunidades del entorno.
Para la elaboración del plan de recuperación del sitio arqueológico, es necesario analizar la distribución de los sectores, el patrón de asentamiento, el manejo de los recursos naturales, las estrategias de control y distribución de espacios de las diferentes culturas que construyeron y ocuparon este sitio (principalmente los Atavillos).

Cabe resaltar que es imprescindible el trabajo en conjunto de un grupo multidisciplinario, que desde diferentes perspectivas pueda contribuir a la elaboración de estrategias para su puesta en valor como destino turístico y así ponerla al servicio de la población canteña.

Este largo proceso necesita de la formulación de una serie de estrategias de trabajo a partir del análisis multidisciplinario previo, lo que no solo se limita al sitio arqueológico, sino al estudio de la población local, su sistema de organización e instituciones.

\section{METAS ESPECÍFICAS}

- Fomentar la investigación y el estudio del sitio arqueológico de Cantamarca, para posicionarlo como destino turístico de la provincia de Canta.

- Incentivar en las autoridades el compromiso y la responsabilidad para la formulación de proyectos turísticos con planes de ejecución a corto plazo.

- Concientizar a la población local sobre la importancia de la recuperación, conservación y preservación de los recursos turísticos culturales y naturales.

- Adecuar la infraestructura de servicios del centro soporte anexo al sitio arqueológico de Cantamarca, para mejorar la calidad de vida de la población y lograr la satisfacción de los turistas durante su visita.

- Contribuir al desarrollo socioeconómico, cultural y sostenible del distrito de Canta.

\section{MARCO TEÓRICO DEL TRABAJO}

Sobre Cantamarca no existen muchas referencias bibliográficas, solo existen dos investigaciones:

La primera, "Las culturas prehispánicas del Departamento de Lima", del autor Pedro Villar Córdova, publicada en el año 1935, es un esfuerzo por identificar y describir los sitios arqueológicos más importantes del departamento de Lima. 
Cantamarca es mencionada por el autor como la "ciudad fortificada" que domina toda la quebrada del Alto Chillón. A partir de su somero reconocimiento del lugar, describe sus características arquitectónicas, destacando los siguientes grupos:

"A) La muralla de circunvalación; B) el acueducto; C) las construcciones incaicas o "Tampus"; D) las construcciones preincaicas; E) la "Pucará" o Atalaya; F) la población militar o casas- tumbas; G) los fuertes de contención; H) las cavernas sepulcrales" (Villar; 1982: 299).

Esta clasificación genérica fue mejorada posteriormente por el arqueólogo Carlos Farfán Lobatón. Cabe señalar que fue este autor quien recogió el término "Kullpi", entre los lugareños para denominar a un tipo particular de estructuras.

El trabajo de Villar Córdova presenta las limitaciones propias de su época, visibles en el uso de ciertas categorías como "ciudad" para un asentamiento que probablemente no alcanzó tales características. Además, la investigación no es lo suficientemente minuciosa, como se hubiese deseado, pues solo realizó breves descripciones, algunos dibujos de planta, corte y fachada de un kullpi.

Sin embargo, recordemos que Villar Córdova no fue un arqueólogo de profesión; contemporáneo de Tello, vivió en los años en que la arqueología apenas si empezaba a desarrollarse. Por ello, pese a las limitaciones del caso, este trabajo ayudará a rescatar la información sobre el sitio, ya que es probable que, desde la época en que el autor visitó Cantamarca hasta la actualidad, haya sufrido el paso del tiempo y la destrucción causada por la mano del hombre.

La segunda investigación, y la más importante, es la desarrollada por el arqueólogo Carlos Farfán Lobatón, quien ha identificado dos zonas. Cantamarca $A$, que a su vez se divide en los sectores I, II, III, IV, V; cuyas características son agrupaciones habitacionales de planta circular u ovalada de aparente jerarquía entre ellas. La otra zona, Cantamarca B, es la denominada zona de 24 colcas rectangulares con orientación de sur a norte.

Farfán realizó trabajos en el sitio en tres temporadas (1984,1987-1988), lo que le permitió mejorar la clasificación realizada por Villar Córdova, planteando sus propias categorías arquitectónicas según la función y el uso: arquitectura habitacional, arquitectura funeraria, arquitectura defensiva, arquitectura vial, arquitectura hidráulica y arquitectura hidráulica.

Se resalta su trabajo pues es el único arqueólogo que ha investigado en el lugar, lo que le ha permitido definir algunas características culturales de Cantamarca, como el haber sido ocupada durante el Intermedio tardío (1100-1470). Asimismo, indica que su fundación debió iniciarse en el sector III y que luego fue expandiéndose, ocupando la pendiente hasta la cumbre del cerro. Así también, plantea que su poder y control en la zona se debió, probablemente, al almacenamiento de excedentes productivos o por el manejo de una economía que incluía la ganadería y la agricultura. Ya para el periodo inca, Cantamarca era considerada un sentamiento estratégico y sede de muchos curacazgos que estaban bajo su control.

El sitio arqueológico de Cantamarca está ubicado al noroeste de la ciudad de Canta (capital de la provincia del mismo nombre en la Región Lima), a escasos $5 \mathrm{~km}$. Sobre la cresta escarpada de uno de los cerros tutelares de la ciudad canteña a 3660 m.s.n.m, en el margen izquierdo del río Chillón.

Antiguamente, era la capital del señorío de Canta, que estaba compuesto por 8 ayllus: Canta, Locha, Carhua, Visca, Lachaqui, Copa, Esquibamba, Caxa Uri. Sus límites territoriales eran: al oeste la Chaupi Yunga (Quives); al este, las pampas de Bombón; al norte, el señorío de los Atavillos; y al sur, los Chaclla (de procedencia Yauyos).

Según la historiadora Dra. María Rostworowski Tovar y el Arqul. Carlos Arturo Farfán Lobatón, Cantamarca fue construida durante el Intermedio tardío (1100d.C.) y sobrevivió a la conquista de los Incas (1450), quienes la reorganizaron y ampliaron hasta el año de 1550 cuando los nuevos conquistadores españoles reubicaron a sus pobladores en la actual ciudad de Canta, siendo Cantamarca abandonada definitivamente. A partir de un somero reconocimiento del lugar, describen sus características arquitectónicas, destacando los siguientes grupos:
A. La muralla de circunvalación;
B. El acueducto;
C. Las construcciones incaicas o "Tampus";
D. Las construcciones preincaicas;
E. La "Pucará" o Atalaya;
F. La población militar o casas- tumbas; 


\section{G. Los fuertes de contención; y \\ H. Las cavernas sepulcrales.}

Cantamarca está construida en la cresta de un cerro escarpado, por lo que son pocos los espacios planos; sus edificios son circulares, con paredes y techos de piedras unidas con barro, sostenidos por una columna central. Para ampliar el espacio hábil se edificaron muchos terraplenes a diferentes niveles, ampliando de este modo el área construida.
Se divide en dos sectores (A-B) diferenciados entre sí por su organización espacial, componentes arquitectónicos y funcionalidad, así como su filiación cultural perteneciente al Intermedio tardío y Horizonte tardío.

La estrategia constructiva en ambas zonas (A y B) consta de paramentos a base de grandes y pequeñas piedras, algunos mampuestos y otros trabajados, unidos a su vez con barro.

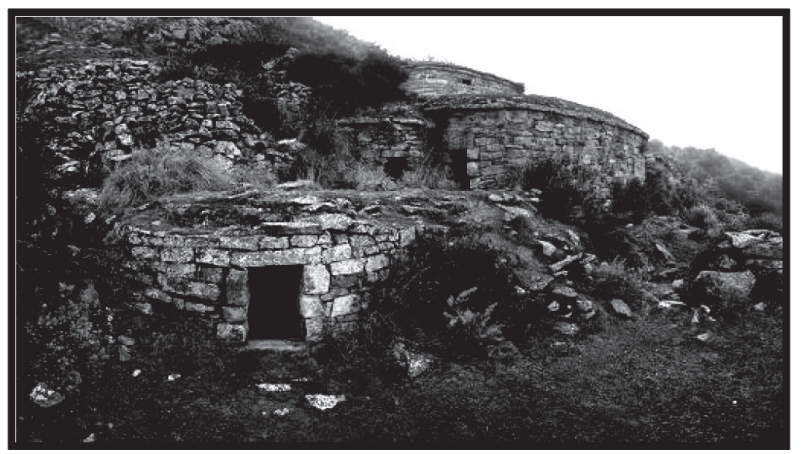

\begin{tabular}{lr}
\hline Un recinto de \\
Cantamarca. De \\
planta circular y \\
columna trapezoidal \\
que sostiene un \\
techo en forma de \\
falsa bóveda.
\end{tabular}
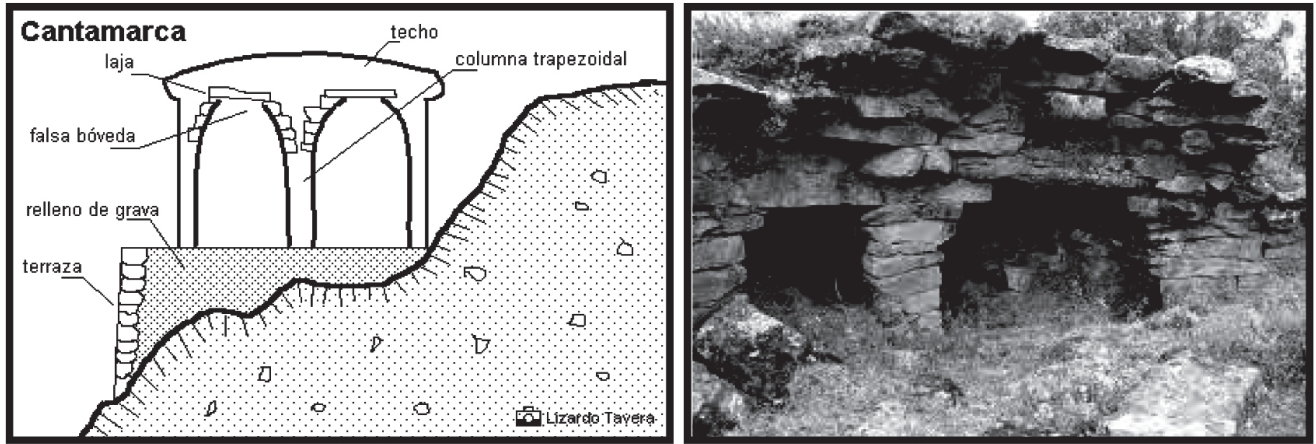

Corte transversal de un recinto ideal en Cantamarca. Se detalla lasprincipales partes. Los muros y el techo son de piedra.

\section{METODOLOGÍAS Y TÉCNICAS DE INVESTIGACIÓN UTILIZADAS}

La investigación realizada por los miembros del equipo Turismo hacia el Bicentenario consta de un diagnóstico situacional del sitio arqueológico, a fin de plantear los procesos y estrategias para desarrollar la puesta en valor del lugar.

La metodología que se utilizó para la obtención de los resultados está en relación con las actividades desarrolladas y aspectos multidisciplinarios que contiene el proyecto.
- Levantamiento de información en los sectores A y B

Para el proceso de registro del sitio arqueológico Cantamarca, se han utilizado las siguientes fichas de registro:

1. Ficha de Registro Estructural - Morfológico del sitio por sectores.

2. Ficha de Registro Fotográfico.

La metodología que se usó para el trabajo de campo fue la siguiente: 
1. Dibujos de corte y perfil del sitio.

2. El registro fotográfico de las excavaciones y de las estructuras de superficie fueron tomadas con cámara digital, registrando cada chullpa en su respectiva ficha.

- Diagnóstico sociocultural de la población

1. Aplicación de encuestas a la población de la localidad de Canta.

2. Desarrollo de entrevistas a las autoridades públicas y pequeños empresarios de establecimientos de hospedaje, restaurantes y de transporte.

3. Descripción y análisis de las festividades y rituales religiosos.

4. Descripción de la gastronomía, tradiciones, y prácticas socioculturales.

- Investigación en archivos del Ministerio de Cultura, Biblioteca de la Nación y Bibliotecas comunales y regionales

1. Identificación de la documentación referente a Cantamarca, existente en los archivos de instituciones públicas.

2. Transcripción paleográfica de la información requerida.

- Procesamiento de información

1. Vaciado de información en equipos digitales.

2. Procesamiento de encuestas con sus variables.

3. Elaboración de cuadros estadísticos.

4. Aplicación del programa SPSS para determinar los datos estadísticos.

- Concientización y capacitación de la población

1. Desarrollo de conferencias y charlas sobre el patrimonio cultural y la importancia de su revalorización.

2. Desarrollo de talleres prácticos.

3. Visitas guiadas a las áreas de investigación.

\section{RESULTADOS}

Proponer los lineamientos y estrategias para la recuperación y puesta en valor del complejo arqueológico de Cantamarca, a partir de investigaciones multidisciplinarias profundas. El resultado será equivalente a un estudio de perfil o expediente técnico para la ejecución integral del proyecto.

\section{Dónde se deben aplicar los resultados de esta} investigación

Se aplicaran en el centro poblado de Canta, provincia de Canta, departamento de Lima. Estos logros traerán como resultado el factor de desarrollo turístico sostenible en la provincia de Canta, distritos y comunidades del entorno.

\section{DISCUSIÓN}

Sobre el complejo arqueológico Cantamarca no existe mucha referencia bibliográfica, solo existen dos investigaciones. La primera, Las culturas prehispánicas del departamento de Lima, de Pedro Villar Córdova, publicada en el año 1935, es un esfuerzo por identificar y describir los sitios arqueológicos más importantes del departamento de Lima. Cantamarca es mencionada por el autor como la "ciudad fortificada" que domina toda la quebrada del Alto Chillón.

A partir de su somero reconocimiento del lugar, describe sus características arquitectónicas, destacando los siguientes grupos:

"A) La muralla de circunvalación; B) el acueducto; C) las construcciones incaicas o "Tampus"; D) Las construcciones preincaicas; E) la "Pucará" o Atalaya; F) la población militar o casas- tumbas; G) los fuertes de contención; H) las cavernas sepulcrales" (Villar; 1982: 299).

Las investigaciones de Villar Córdova presenta las limitaciones propias de su época, visibles en el uso de ciertas categorías como "ciudad" para un asentamiento que probablemente no alcanzó tales características. Además, no es lo suficientemente minucioso, solo realizó breves descripciones, algunos dibujos de planta, corte y fachada de un kullpi.

Sin embargo, recordemos que Villar Córdova no fue un arqueólogo de profesión; contemporáneo de Tello, vivió en los años en que la arqueología apenas empezaba a desarrollarse. Por ello, pese a las limitaciones del caso, este trabajo nos ayuda a rescatar la información sobre el sitio, ya que es probable que, desde la época en que el autor visitó Cantamarca hasta la actualidad, haya sufrido el paso del tiempo, agregado a la destrucción causada por la mano del hombre.

\section{CONCLUSIONES}

1. Se ha hallado que algunos recintos poseen un buen estado de conservación; sin embargo, la gran mayoría presenta derrumbes, roturas 
y techos caídos. La vegetación, los musgos y los líquenes han cubierto los muros interiores y exteriores de los recintos, representando un grave peligro; las raíces de estos se incrustan en la pared o en las bases de los muros y los debilitan hasta que se desplomen.

2. El agua es otro elemento que causa daño, ya sea generada por la lluvia o por la humedad que traen consigo las neblinas, terminará por debilitar las construcciones a base de piedra y argamasa. Y por último, el agente más dañino: el hombre. Observamos que muchas de las unidades habitacionales presentan signos de huaqueo, acto deleznable que ocasiona la destrucción de muros o contextos funerarios en busca de objetos de valor, cuya consecuencia además es la remoción de tierra, piedras y materiales arqueológicos que ya descontextualizados no sirven para ninguna investigación y, por ende, no aportarán ningún conocimiento sobre el pasado.

3. De todos los sectores recorridos, el sector III y IV son los que en mejor estado de conservación están. Los demás sectores, sobre todo el I y el V, presentan pocos muros en pie; los agentes naturales y el hombre, mediante huaqueos o peregrinaciones religiosas, han contribuido a su destrucción.

\section{AGRADECIMIENTOS}

Al Dr. Bernardino Ramírez Bautista, vicerrector de Investigación de la UNMSM, por fomentar la investigación científica de docentes y estudiantes en el aspecto histórico, arqueológico y turístico, para lo cual se realizó los estudios de la Estrategia de la Puesta en Valor del Sitio Arqueológico Cantamarca para el Desarrollo del Turismo Sostenible en la Provincia de Canta.

\section{REFERENCIAS BIBLIOGRÁFICAS}

CASAVERDE RÍOS, Guido (2007) Ficha Técnica: Cantamarca A Y B. Ministerio de Cultura, Lima.

FARFÁN LOBATÓN, Carlos (2011) "Arquitectura prehispánica de Cantamarca-Canta” en Arquitectura prehispánica tardía: construcción y poder en los Andes centrales. Fondo Editorial UCSS y Universidad Católica Sedes Sapientiae, 1 ra edición.

HORKHEIMER, Hans (1965) "Identificación y Bibliografía de Importantes Sitios Prehispánicos del Perú" En: Arqueológicas № 8 M.N.A.A. Lima.
ROSTOROWSKI DE DIEZ CANSECO, María. (1978)

"Señoríos Indígenas de Lima y Canta”.IEP. Lima 280 pg.

VILLAR CÓRDOVA, Pedro (1935) "Arqueologia del Departamento de Lima" Ed. Atusparia, $2^{\circ}$ edic. Lima 1982.

\section{ANEXOS}

- Planos
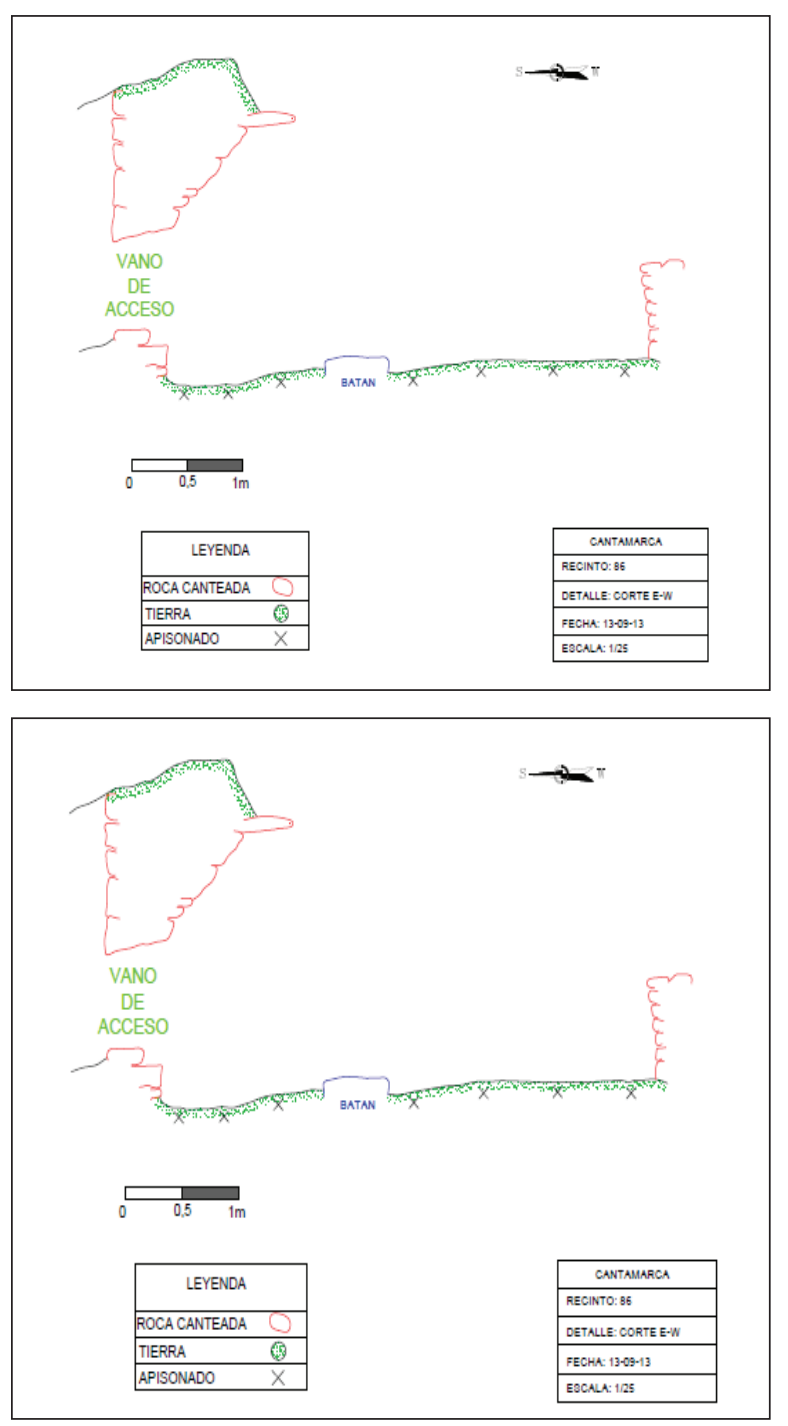

Fichas Técnicas Arqueológicas

\section{FICHA №1}

UBICACIÓN: Catamarca A

SECTOR: I

\section{NÚMERO DE RECINTO: 8}

\section{REGISTRO}

1. Tipo de estructura: Recinto de planta rectangular 
2. Tamaño (largo, ancho, altura: para todos el máximo):

Largo: 14.60

Ancho: 5.90

Alto: 2.00

3. Material de construcción: Piedra

4. Técnica de construcción: Mampostería

5. Condición o estado de conservación: En mal estado de conservación. Presenta techumbre caída. Muros este, oeste y sur están derrumbados. Asimismo, la vegetación crece desmedidamente.

6. Cimientos y bases (descripción, medios involucrados en su destrucción): Aunque aparentemente aún han resistido al tiempo, la humedad poco a poco va debilitándolos.

7. Pavimentos y pisos (descripción, natural, preparado, técnica, medios involucrados en su destrucción): El piso no se puede observar debido al polvo, piedras caídas.
8. Evidencia de cubierta (descripción, tipo, técnica, medios involucrados en su destrucción): No presenta ningún tipo de cubierta, se ha desplomado.

9. Acceso y vanos (lados abiertos, puertas, ventanas, pasadizos: ubicación, tipo, dimensiones, forma, medios involucrados en su destrucción): No presenta vanos de acceso conservados.

10.Elementos arquitectónicos menores (rellenos, nichos, etc.: ubicación, tipo, dimensiones, forma, medios involucrados en su destrucción): no presenta nichos.

11.Detalle mediante fotos: Ver fotos 3453

\section{COMENTARIO}

Este sector es el más afectado por el ganado vacuno que ingresa al interior de los recintos en busca de vegetación.

FECHA DEL REGISTRO: 28/12/13 